\title{
Characterization of a PET Camera Optimized for Prostate Imaging
}

\author{
J. S. Huber, Member, IEEE, W. S. Choong, Member, IEEE, W. W. Moses, Senior Member, IEEE, J. Qi, \\ Member, IEEE, J. Hu, Member, IEEE, G. C. Wang, Member, IEEE, D. Wilson, S. Oh, R. H. Huesman, Fellow, \\ IEEE, S. E. Derenzo, Fellow, IEEE
}

\begin{abstract}
We present the characterization of a positron emission tomograph for prostate imaging that centers a patient between a pair of external curved detector banks (ellipse: $45 \mathrm{~cm}$ minor, $70 \mathrm{~cm}$ major axis). The distance between detector banks adjusts to allow patient access and to position the detectors as closely as possible for maximum sensitivity with patients of various sizes. Each bank is composed of two axial rows of 20 $\mathrm{HR}+$ block detectors for a total of 80 detectors in the camera. The individual detectors are angled in the transaxial plane to point towards the prostate to reduce resolution degradation in that region. The detectors are read out by modified HRRT data acquisition electronics. Compared to a standard whole-body PET camera, our dedicated-prostate camera has the same sensitivity and resolution, less background (less randoms and lower scatter fraction) and a lower cost. We have completed construction of the camera. Characterization data and reconstructed images of several phantoms are shown. Sensitivity of a point source in the center is $946 \mathrm{cps} / \mu \mathrm{Ci}$. Spatial resolution is $4 \mathrm{~mm}$ FWHM in the central region.
\end{abstract}

\section{INTRODUCTION}

$\mathrm{W}$ E present the characterization of a positron tomograph optimized to image the prostate. This camera is designed to provide an efficient, sensitive, and relatively inexpensive instrument that can be used to detect early stage prostate cancer (to confirm elevated prostate specific antigen levels), guide biopsy and treatment decisions, monitor response to therapy, and detect local reoccurrence [1]. Initially $\left[{ }^{11} \mathrm{C}\right]$ choline will be used as the PET tracer for imaging prostate tumors [2]. There are also several ${ }^{18} \mathrm{~F}$ radiopharmaceuticals currently under investigation for prostate cancer imaging that are expected to have increased commercial viability, including $\left[{ }^{18} \mathrm{~F}\right]$ fluorocholine [3], and these may be used in the future.

Coincidence imaging of positron emitters is achieved using a pair of external curved detector banks with the patient centered between them. The two banks form an incomplete elliptical ring of detectors with a $45 \mathrm{~cm}$ minor axis and a 70 $\mathrm{cm}$ major axis. Figure 1a shows the transaxial view of the

Manuscript received October 11, 2005. This work was supported in part by the Director, Office of Science, Office of Biological and Environmental Research, Medical Science Division, U.S. Department of Energy under Contract No. DE-AC02-05CH11231, in part by Department of Defense grant number DAMD17-02-1-0081, and in part by National Institute for Biomedical Imaging and Bioengineering grant numbers R01-EB-00194 and R01-HL-071253.

J.S. Huber, W.S. Choong, W. W. Moses, J. Hu, G.. C. Wang, D. Wilson, S. Oh, R. H. Huesman and S. E. Derenzo are with the Lawrence Berkeley National Laboratory, Mailstop 55-121, 1 Cycltron Road, Berkeley, CA 94720 USA (telephone: 510-486-6445, e-mail: jshuber@lbl.gov).

J. Qi is with the Department of Biomedical Engineering, University of California, Davis, CA 95616 USA (telephone: 530-754-6142, e-mail: qi@ucdavis.edu). camera. Each bank consists of two axial rows of $20 \mathrm{HR}+$ [4] PET block detector modules for a total of 80 detectors per camera; thus the camera uses about one-quarter the number of detectors as an EXACT HR or HR+ scanner which reduces our camera cost. Our dedicated-prostate camera has a reduced axial extent and thus better shielding than a conventional whole body PET scanner, which reduces the number of scatter and random events. The average distance to our detectors is about one-half that of a conventional PET system. Hence, we achieve lower backgrounds and improved detection efficiency in the central imaging volume at a lower cost.

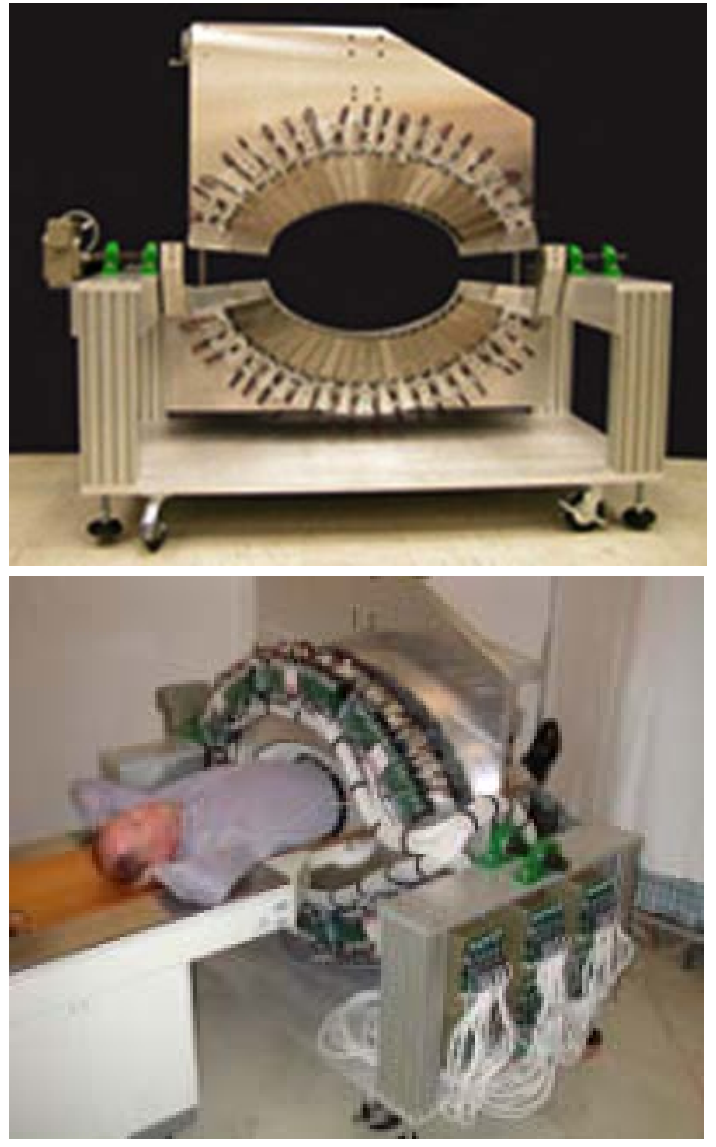

Fig. 1. (a) Photograph of the partially-assembled camera with the lead shielding on one side removed and a single axial row of detector modules visible. The individual detector modules are angled to point towards the center of the camera (where the prostate will be positioned). (b) Photograph of the completed camera with a person in position on the patient table.

The detectors are read out by modified HRRT data acquisition electronics [5]. The individual detector modules are angled to point towards the camera center near the prostate location, thus reducing penetration effects for annihilation 
photons originating in the prostate. Reconstruction of annihilation photons from tissues outside the prostate bed will be less precise due to penetration (astigmatism) effects.

The camera design includes inter-module septa that extend 5 $\mathrm{cm}$ beyond the scintillator crystals to reduce background events from random coincidences and from photons that Compton scatter in the patient [6]. However, all data presented in this paper were taken with the completed camera in 3D mode (i.e., without septa), as shown in Figure 1b. The camera was calibrated using semi-automated software with crystalspecific energy cuts. A $300 \mathrm{keV}$ energy threshold and $10 \mathrm{nsec}$ coincidence timing window were used for all measurements.

\section{Count Rates And Sensitivity}

The count rates as a function of activity density were measured using a $19 \mathrm{~cm}$ diameter by $20 \mathrm{~cm}$ long cylindrical phantom filled with ${ }^{11} \mathrm{C}$ solution and centered in the camera. Data were collected for 10 seconds every 3 minutes. Figure 2 shows the count rate as a function of activity density for total, prompt, random and true+scatter coincident events. The maximum total count rate is $528 \mathrm{khz}$ at an activity density of $1.5 \mu \mathrm{Ci} / \mathrm{ml}$. The true + scatter events cross the randoms at 0.41 $\mu \mathrm{Ci} / \mathrm{ml}$.

Coincidence event rates were measured by scanning a 1.5 $\mathrm{mm}$ diameter $47 \mu \mathrm{Ci}{ }^{68} \mathrm{Ge}$ point source along the central axis of the patient port in $3 \mathrm{~mm}$ steps. Figure 3 shows the system sensitivity as a function of axial position. The peak absolute sensitivity is $946 \mathrm{cps} / \mu \mathrm{Ci}(2.6 \%)$ in $3 \mathrm{D}$ mode. The system sensitivity at the center of the field of view (i.e., position 44 $\mathrm{mm}$ ) is the same as an EXACT HR in 3D mode, and 8 times higher than the HR in 2D mode [7].

(a)

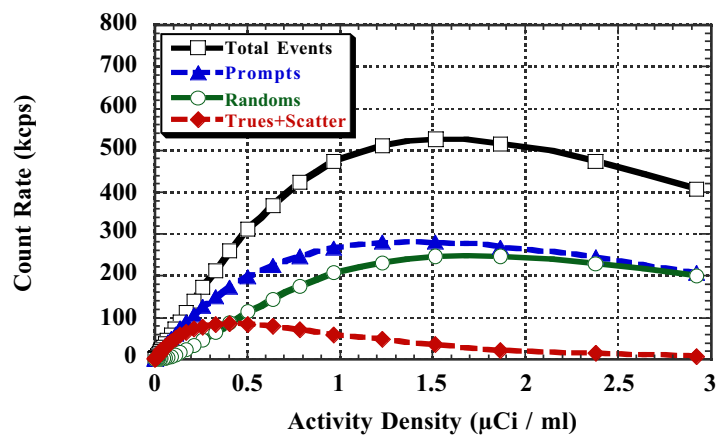

(b)

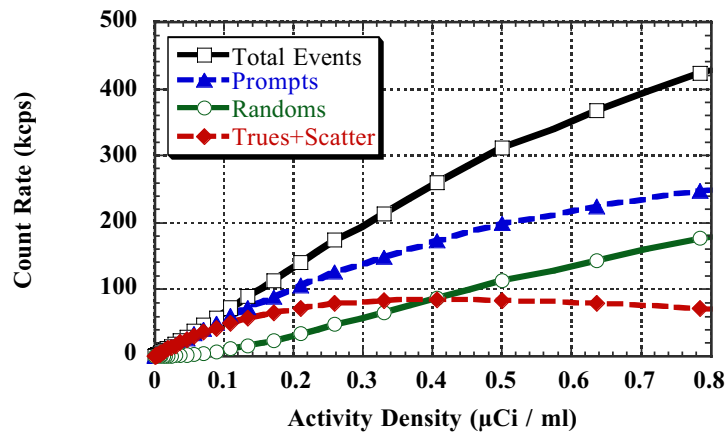

Fig. 2. Count rates as a function of ${ }^{11} \mathrm{C}$ activity density for total, prompt, random and true + scatter coincident events acquired with a cylinder phantom (19 $\mathrm{cm}$ diameter, $20 \mathrm{~cm}$ long) centered in the camera. Plots show two different ranges of activity density.

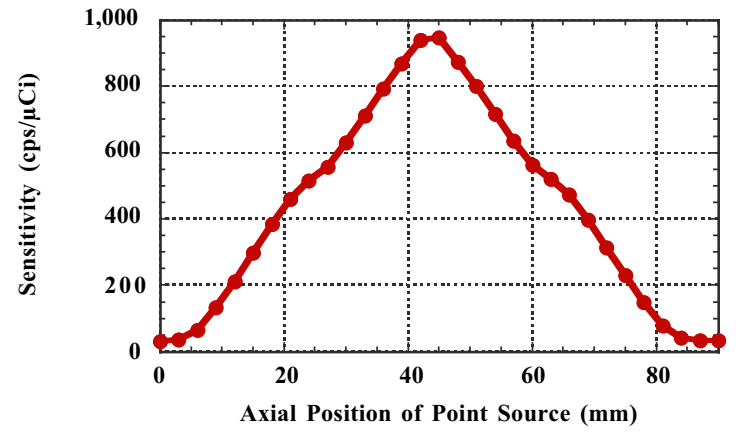

Fig. 3. System sensitivity as a function of axial position, using a $47 \mu \mathrm{Ci}$ ${ }^{68} \mathrm{Ge}$ point source.

\section{RECONSTRUCTED IMAGES}

(a)

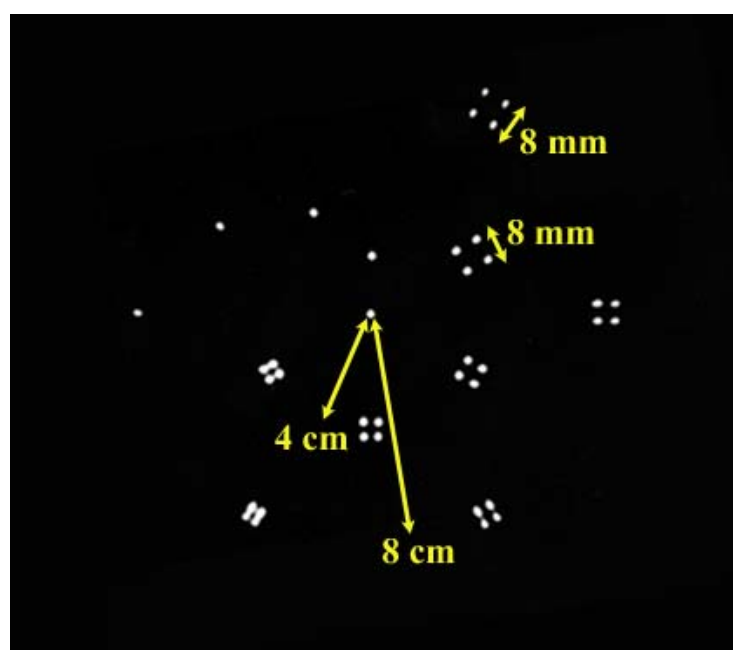

(b)

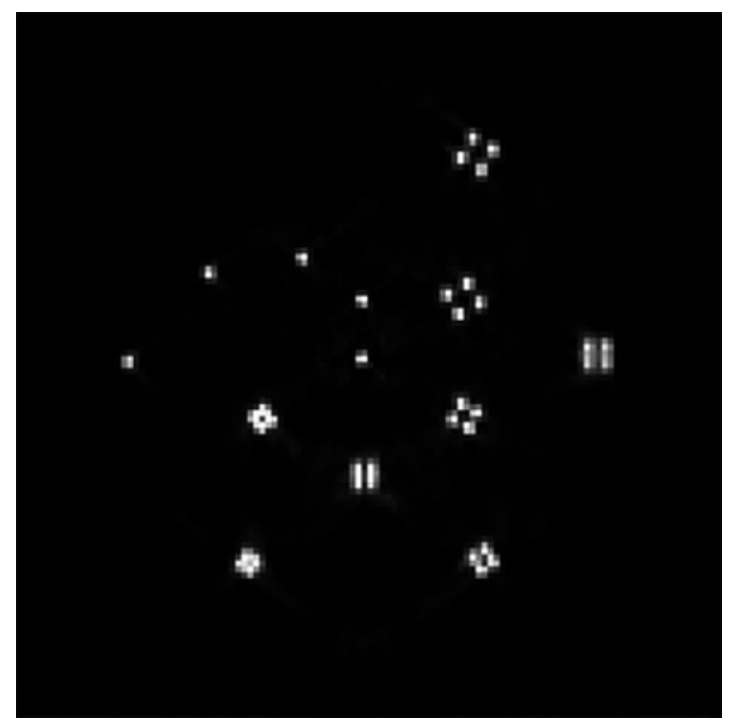

Fig. 4. (a) 37-line source phantom, with each line $5 \mathrm{~cm}$ long in the axial direction. In the transverse plane, the single line sources are $2,4,6$, and 8 $\mathrm{cm}$ from the central line. Clusters of four line sources are placed radially at 4 and $8 \mathrm{~cm}$ from the central line. The four line clusters are spaced $8,6,5$, and $4 \mathrm{~mm}$ apart. (b) Reconstructed image of the phantom. The central line was placed in the center of the camera. Image represents $45 \mathrm{M}$ counts. Voxel size equals $2 \mathrm{~mm} \times 2 \mathrm{~mm} \times 2 \mathrm{~mm}$.

We have developed a 3D iterative penalized maximum likelihood reconstruction algorithm that is very flexible in modeling arbitrary scanner geometry [8]. The projection 
matrix models the exact position and orientation of the detector blocks and crystal penetration effect. The attenuation correction factors are calculated based on the measured phantom geometry and uniform attenuation coefficients. A voxel size of $2 \mathrm{~mm} \times 2 \mathrm{~mm} \times 2 \mathrm{~mm}$ is used. The randoms background is estimated and subtracted using the standard delayed window technique. A rectangular planar phantom 69 $\mathrm{cm} \times 10 \mathrm{~cm} \times 1 \mathrm{~cm}$ is used for normalization. Coincident data are acquired with the planar phantom centered between the detector banks (i.e., placed horizontally in Fig. 1a). The data are used to correct for the efficiency of the lines of response between detector banks.

Figure $4 \mathrm{a}$ shows a 37-line source phantom. Single line sources are placed 2, 4, 6 and $8 \mathrm{~cm}$ from the central line. Clusters of four line sources are placed radially at 4 and $8 \mathrm{~cm}$ from the central line, and the line sources in each cluster are spaced 8, 6, 5 and $4 \mathrm{~mm}$ apart (in clockwise order from the 8 $\mathrm{mm}$ labeled clusters in Fig. 4a). Figure $4 \mathrm{~b}$ shows the reconstructed image of this 37-line source phantom when filled with ${ }^{18} \mathrm{~F}$ solution at an initial activity of $0.8 \mathrm{mCi}$ and imaged for 2 hours. Only minor blurring is seen for line sources placed further from the center, demonstrating good resolution in the $16 \mathrm{~cm}$ diameter central region. We are able to resolve line sources that are separated by $5 \mathrm{~mm}$ when placed at a diameter of $8 \mathrm{~cm}$ and $16 \mathrm{~cm}$.

(b)
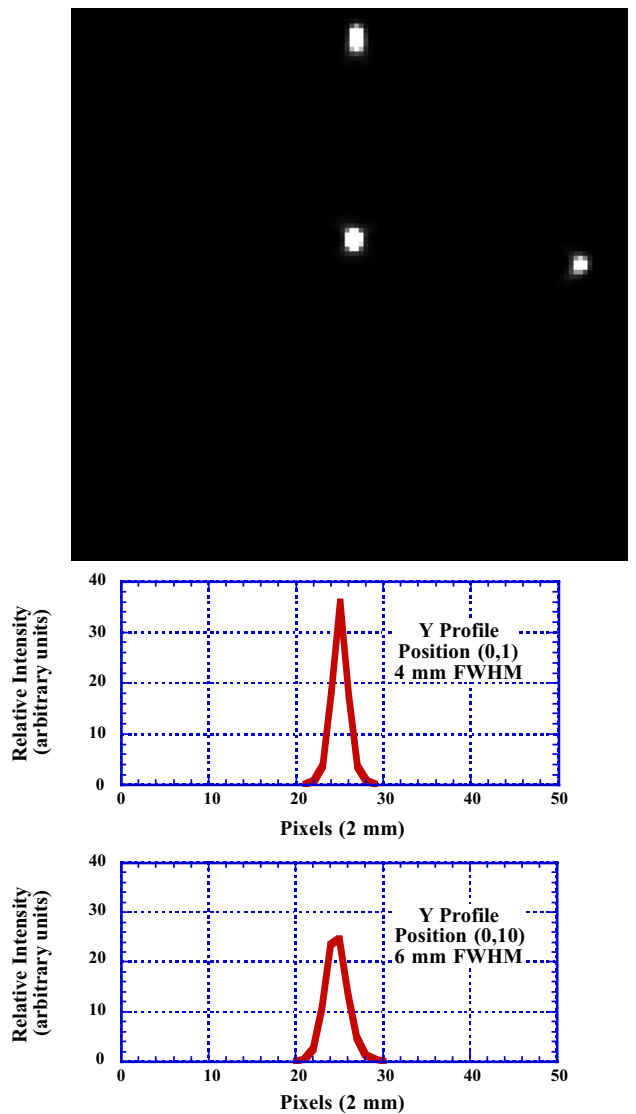

Fig. 5. (a) Combined reconstructed images of an uniform background and a 20 -gauge needle $\left(0.6 \mathrm{~mm}\right.$ ID) filled with a $34 \mu \mathrm{Ci}{ }^{18} \mathrm{~F}$ solution at three different positions. Relative to the camera center, the needle was imaged at transverse positions $(0,1 \mathrm{~cm}),(0,10 \mathrm{~cm})$ and $(10 \mathrm{~cm}, 0)$. (b) Profile in the $y$ direction for needle positioned at $(0,1 \mathrm{~cm})$ and $(0,10 \mathrm{~cm})$. Image represents $13 \mathrm{M}$ events per position. Voxel size equals $2 \mathrm{~mm} \times 2 \mathrm{~mm} \times 2 \mathrm{~mm}$. (a)

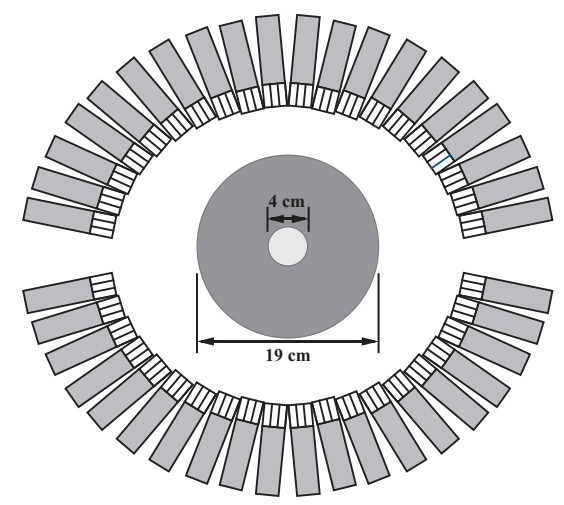

(b)
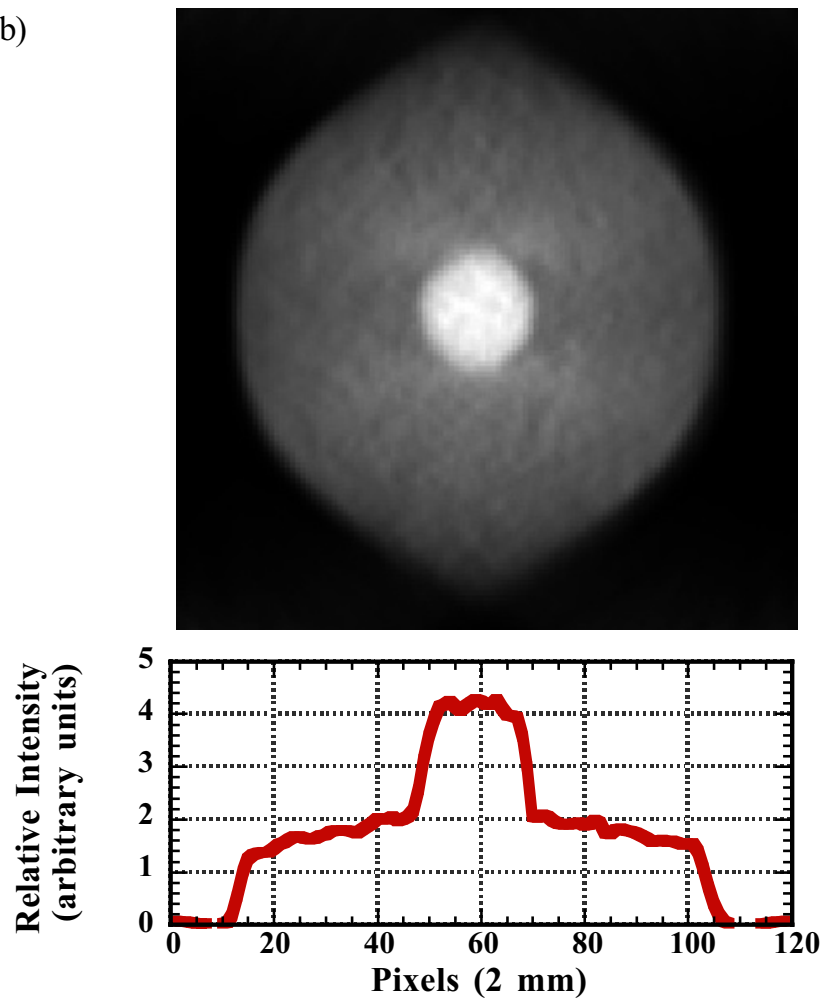

Fig. 6. (a) Concentric-cylinder phantom with three times higher activity density in the inner cylinder than the outer cylinder cavity. (b) Reconstructed image of phantom and profile. Initial ${ }^{18} \mathrm{~F}$ activity density was $0.04 \mu \mathrm{Ci} / \mathrm{ml}$ in the outer cavity and $0.11 \mu \mathrm{Ci} / \mathrm{ml}$ in inner. Imaging time was 1 hour. Image represents $68 \mathrm{M}$ counts. Voxel size equals $2 \mathrm{~mm}$ x $2 \mathrm{~mm}$ x $2 \mathrm{~mm}$. No scatter correction was performed.

Spatial resolution was measured by imaging a 1 -inch 20 gauge needle filled with $34 \mu \mathrm{Ci}^{18} \mathrm{~F}$ solution at three positions in the field of view. Relative to the camera center, the needle was imaged at transverse positions $(0,1 \mathrm{~cm}),(0,10 \mathrm{~cm})$ and $(10 \mathrm{~cm}, 0)$ in accordance with the NEMA standard [9]. Figure 5a shows the combined reconstructed images. The transverse spatial resolution at $1 \mathrm{~cm}$ is $4 \mathrm{~mm}$ full width at half maximum (FWHM). At $10 \mathrm{~cm}$, the average transverse radial resolution is $5.5 \mathrm{~mm} \mathrm{FWHM}$ and average transverse tangential resolution is $4 \mathrm{~mm}$ FWHM. Figure $5 \mathrm{~b}$ shows the profile in the $\mathrm{y}$ direction for the needle positioned at $(0,1 \mathrm{~cm})$ and $(0$, $10 \mathrm{~cm}$ ); only minor blurring is seen for the point source placed $10 \mathrm{~cm}$ from the center. Ideally a filtered back projection reconstruction would be used when measuring the spatial resolution, as specified by the NEMA standard, but this is difficult for our geometry. Instead, we added an uniform 
background to the point source data set and reconstructed with our standard iterative algorithm. However, an insufficient amount of background was added, so the non-negativity constraint was still active.

Figure 6a shows a concentric-cylinder phantom centered between the detector banks. The outer compartment has a diameter of $19 \mathrm{~cm}$, the inner has a diameter of $4 \mathrm{~cm}$, and both have a length of $20 \mathrm{~cm}$. The inner and outer compartments of the phantom were filled with ${ }^{18} \mathrm{~F}$ solution at an uniform initial activity density of $0.11 \mu \mathrm{Ci} / \mathrm{ml}$ and $0.04 \mu \mathrm{Ci} / \mathrm{ml}$, respectively. Thus the inner compartment had three times higher activity density than the outer. For comparison, the expected activity density for humans is about $0.13 \mu \mathrm{Ci} / \mathrm{ml}$ and a human prostate has a diameter of about $5 \mathrm{~cm}$. Figure $6 \mathrm{~b}$ shows the reconstructed image of this phantom when imaged for 1 hour, as well as the horizontal profile through the center. The image represents $68 \mathrm{M}$ counts. No scatter correction was performed. We are able to reconstruct the simple prostate phantom with only minor artifacts in the uniform "body" distribution. Artifacts at the top and bottom of the image are due to incomplete sampling from the detector bank gaps, and these artifacts will be reduced when we include events that are coincident within the same detector bank.

Figure 7a shows the NEMA body phantom [10]. A "lung" cylinder insert is filled with foam beads and cold water, then placed in the center of the phantom. It is surrounded by six spheres that are placed on a $6 \mathrm{~cm}$ radius in the transaxial plane with a common axial center line. The four smallest spheres were filled with an initial ${ }^{18} \mathrm{~F}$ activity density of $3.5 \mu \mathrm{Ci} / \mathrm{ml}$. The torso and two largest spheres were filled with cold water. Figure $7 \mathrm{~b}$ shows the reconstructed transaxial and sagittal images. The fill tube of the spheres are visible in the sagittal images. We can resolve all four of the "hot" spheres.

(a)

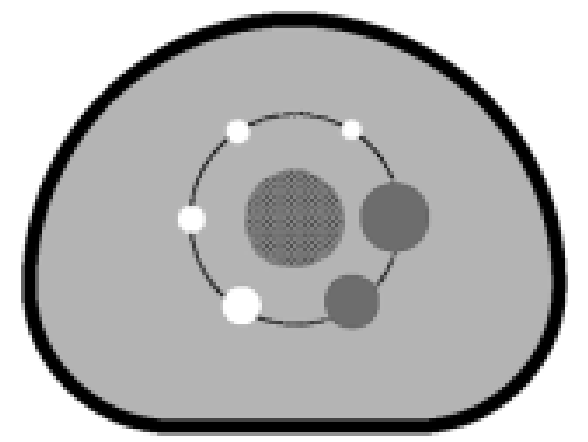

(b)
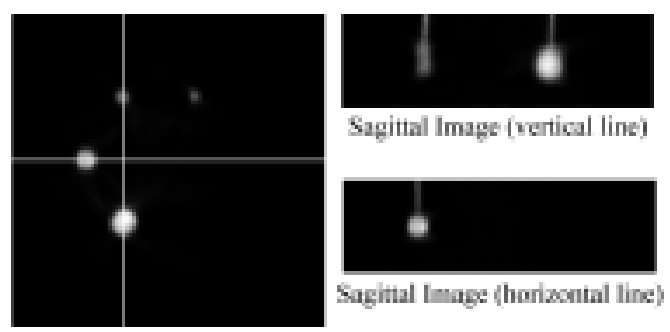

Transaxial Image

Fig. 7. (a) NEMA body phantom. Four smallest spheres filled with initial ${ }^{18} \mathrm{~F}$ activity density of $3.5 \mu \mathrm{Ci} / \mathrm{ml}$. Lung insert (in center), torso and two largest spheres filled with cold water. Sphere inner diameters are $37 \mathrm{~mm}, 28$ $\mathrm{mm}, 22 \mathrm{~mm}, 17 \mathrm{~mm}, 13 \mathrm{~mm}$, and $10 \mathrm{~mm}$. (b) Reconstructed images of phantom when centered in the camera (with spheres also centered axially). Imaging time was 1 hour and images represent $25 \mathrm{M}$ counts. Voxel size equals $2 \mathrm{~mm} \times 2 \mathrm{~mm} \times 2 \mathrm{~mm}$.

\section{CONCLUSIONS}

We have designed and built a positron emission tomograph optimized for prostate imaging. The sensitivity of a point source in the center is $946 \mathrm{cps} / \mu \mathrm{Ci}$, equivalent to the EXACT $\mathrm{HR}$ in 3D mode. Using a $19 \mathrm{~cm}$ diameter cylindrical phantom, the maximum total count rate is $528 \mathrm{khz}$ at 1.5 $\mu \mathrm{Ci} / \mathrm{ml}$ and the trues + scatter events cross the randoms at 0.41 $\mu \mathrm{Ci} / \mathrm{ml}$. We have reconstructed images of line sources, and the spatial resolution is $4 \mathrm{~mm}$ FWHM in the central region. We have successfully reconstructed images of extended simple prostate and NEMA body phantoms. We plan to complete NEMA characterization of the camera, then move on to patient studies. We plan to develop dual-modality PET/Ultrasound imaging of the prostate.

\section{ACKNOWLEDGMENT}

This work was supported in part by the Director, Office of Science, Office of Biological and Environmental Research, Medical Science Division, U.S. Department of Energy under Contract No. DE-AC02-05CH11231, in part by Department of Defense grant number DAMD17-02-1-0081, and in part by National Institute for Biomedical Imaging and Bioengineering grant numbers R01-EB-00194 and R01-HL-071253.

\section{REFERENCES}

[1] J.S. Huber, S.E. Derenzo, J. Qi, et al., "Conceptual Design of a Compact Positron Tomograph for Prostate Imaging," IEEE Trans. Nucl. Sci., vol. 48, pp. 1506-1511, 2001.

[2] T. Hara, N. Kosaka, and H. Kishis, "PET imaging of prostate cancer using carbon-11-choline," J Nucl Med, vol. 39 (Suppl), pp. 250P, 1997.

[3] T.R. Degrado, R.E. Coleman, S.W. Baldwin, et al., "Fluorine-18 fluorocholine $(\mathrm{FCH})$ as an oncological PET tracer: evaluation in murine prostate cancer xenograft model," J Nucl Med, vol. 41 (Suppl), pp. 231, 2000 .

[4] B.Bendriem, M.Casey, M.Dahlbom, et al., "Evaluation of the ECAT EXACT HR+: a new positron camera with 2D/3D acquisition capabilities and nearly isotropic spatial resolution," J. Nucl. Med., vol. 37, no. 5, pp. 170P, 1996.

[5] W.W. Moses, J.W. Young, K. Baker, et al, "The Electronics System for the LBNL Positron Emission Mammography (PEM) Camera," IEEE Trans. Nucl. Sci., vol. 48, pp. 632-636, 2001

[6] J. Qi, J.S. Huber, R. H. Huesman, et al., "Septa Design for a Prostate Specific PET Camera," IEEE Trans. Nucl. Sci., vol. 52, pp. 107-113, 2005 .

[7] K. Wienhard, M. Dahlbom, L. Eriksson, et al., "The ECAT EXACT HR: Performance of a New High Resolution Positron Scanner," $J$ Comput Assist Tomogr, vol. 18, pp. 110-118, 1994.

[8] J. Hu, J. Qi, J. Huber, et al., "MAP image reconstruction for arbitrary geometry PET system with application to a prostate-specific scanner," Proceedings of International Meeting on Fully $3 D$ Image Reconstruction in Radiology and Nuclear Medicine, pp. 416-420, Salt Lake, Utah, 2005.

[9] "Performance Measurements of Positron Emission Tomographs." NEMA Standards Publication NU 2-2001, National Electrical Manufacturers Association (NEMA), Washington, D.C., 2001.

[10] Data Spectrum Corporation, Hillsborough, NC 27278. 